\title{
Existence and Asymptotic Behavior of Positive Solutions of Functional Differential Equations of Delayed Type
}

\author{
J. Diblík and M. Kúdelčíková \\ Department of Mathematics, Faculty of Science, University of Žilina, 01026 Žilina, Slovakia \\ Correspondence should be addressed to J. Diblík, josef.diblik@fpv.uniza.sk
}

Received 30 September 2010; Accepted 14 October 2010

Academic Editor: Elena Braverman

Copyright (C 2011 J. Diblík and M. Kúdelčíková. This is an open access article distributed under the Creative Commons Attribution License, which permits unrestricted use, distribution, and reproduction in any medium, provided the original work is properly cited.

Solutions of the equation $\dot{y}(t)=-f\left(t, y_{t}\right)$ are considered for $t \rightarrow \infty$. The existence of two classes of positive solutions which are asymptotically different is proved using the retract method combined with Razumikhin's technique. With the aid of two auxiliary linear equations, which are constructed using upper and lower linear functional estimates of the right-hand side of the equation considered, inequalities for both types of positive solutions are given as well.

\section{Introduction}

Let $C\left([a, b], \mathbb{R}^{n}\right)$, where $a, b \in \mathbb{R}, a<b$, be the Banach space of the continuous mappings from the interval $[a, b]$ into $\mathbb{R}^{n}$ equipped with the supremum norm

$$
\|\psi\|_{C}=\sup _{\theta \in[a, b]}\|\psi(\theta)\|, \quad \psi \in C\left([a, b], \mathbb{R}^{n}\right),
$$

where $\|\cdot\|$ is the maximum norm in $\mathbb{R}^{n}$. In the case of $a=-r<0$ and $b=0$, we will denote this space as $C_{r}^{n}$, that is,

$$
C_{r}^{n}:=C\left([-r, 0], \mathbb{R}^{n}\right)
$$

If $\sigma \in \mathbb{R}^{n}, A \geq 0$, and $y \in C\left([\sigma-r, \sigma+A], \mathbb{R}^{n}\right)$, then, for each $t \in[\sigma, \sigma+A]$, we define $y_{t} \in C_{r}^{n}$ by $y_{t}(\theta)=y(t+\theta), \theta \in[-r, 0]$. 
The present article is devoted to the problem of the existence of two classes of asymptotically different positive solutions of the delayed equation

$$
\dot{y}(t)=-f\left(t, y_{t}\right)
$$

for $t \rightarrow+\infty$, where $f: \Omega \rightarrow \mathbb{R}$ is a continuous quasibounded functional that satisfies a local Lipschitz condition with respect to the second argument and $\Omega$ is an open subset in $\mathbb{R} \times C_{r}^{1}$ such that conditions which use $f$ are well defined.

The main supposition of our investigation is that the right-hand side of (1.3) can be estimated as follows:

$$
C_{A}(t) y_{t}(-r) \leq f\left(t, y_{t}\right) \leq C_{B}(t) y_{t}(-r)
$$

where $\left(t, y_{t}\right) \in \Omega$, and $C_{A}, C_{B}:\left[t_{0}-r, \infty\right) \rightarrow \mathbb{R}^{+}:=(0, \infty), t_{0} \in \mathbb{R}$ are continuous functions satisfying

$$
\begin{aligned}
0<C_{A}(t) \leq & C_{B}(t) \leq \frac{1}{(r e)}, \quad t \in\left[t_{0}-r, \infty\right), \\
& \int_{t_{0}-r}^{\infty} C_{B}(t) \mathrm{d} t<1 .
\end{aligned}
$$

Quite lots of investigations are devoted to existence of positive solutions of different classes of equations (we mention at least monographs [1-6] and papers [7-12]). The investigation of two classes of asymptotically different solutions of (1.3) has been started in the paper [13] using a monotone iterative technique and a retract principle. Assumptions of results obtained are too cumbersome and are applied to narrow classes of equations. In the presented paper we derive more general statements under weaker conditions. This progress is related to more general inequalities (1.4) for the right-hand side of (1.3) which permit to omit utilization of properties of solutions of transcendental equations used in [13].

\subsection{Ważewski's Principle}

In this section we introduce Ważewski's principle for a system of retarded functional differential equations

$$
\dot{y}(t)=F\left(t, y_{t}\right),
$$

where $F: \Omega^{*} \mapsto \mathbb{R}^{n}$ is a continuous quasibounded map which satisfies a local Lipschitz condition with respect to the second argument and $\Omega^{*}$ is an open subset in $\mathbb{R} \times \mathrm{C}_{r}^{n}$. We recall that the functional $F$ is quasibounded if $F$ is bounded on every set of the form $\left[t_{1}, t_{2}\right] \times C_{r L}^{n} \subset$ $\Omega^{*}$, where $t_{1}<t_{2}, C_{r L}^{n}:=C([-r, 0], L)$ and $L$ is a closed bounded subset of $\mathbb{R}^{n}$ (compare [2, page 305]).

In accordance with [14], a function $y(t)$ is said to be a solution of system (1.7) on [ $\sigma-$ $r, \sigma+A)$ if there are $\sigma \in \mathbb{R}$ and $A>0$ such that $y \in C\left([\sigma-r, \sigma+A), \mathbb{R}^{n}\right),\left(t, y_{t}\right) \in \Omega^{*}$, and $y(t)$ satisfies the system (1.7) for $t \in[\sigma, \sigma+A)$. For a given $\sigma \in \mathbb{R}, \varphi \in C$, we say $y(\sigma, \varphi)$ is a solution 
of the system (1.7) through $(\sigma, \varphi) \in \Omega^{*}$ if there is an $A>0$ such that $y(\sigma, \varphi)$ is a solution of the system (1.7) on $[\sigma-r, \sigma+A)$ and $y_{\sigma}(\sigma, \varphi)=\varphi$. In view of the above conditions, each element $(\sigma, \varphi) \in \Omega^{*}$ determines a unique solution $y(\sigma, \varphi)$ of the system (1.7) through $(\sigma, \varphi) \in \Omega^{*}$ on its maximal interval of existence $I_{\sigma, \varphi}=[\sigma, a), \sigma<a \leq \infty$ which depends continuously on initial data [14]. A solution $y(\sigma, \varphi)$ of the system (1.7) is said to be positive if

$$
y_{i}(\sigma, \varphi)>0
$$

on $[\sigma-r, \sigma] \cup I_{\sigma, \varphi}$ for each $i=1,2, \ldots, n$. A nontrivial solution $y(\sigma, \varphi)$ of the system (1.7) is said to be oscillatory on $I_{\sigma, \varphi}$ (under condition $I_{\sigma, \varphi}=[\sigma, \infty)$ ) if $(1.8)$ does not hold on any subinterval $\left[\sigma_{1}, \infty\right) \subset[\sigma, \infty), \sigma_{1} \geq \sigma$.

As a method of proving the existence of positive solutions of (1.3), we use Ważewski's retract principle which was first introduced by Ważewski [15] for ordinary differential equations and later extended to retarded functional differential equations by Rybakowski [16] and which is widely applicable to concrete examples. A summary of this principle is given below.

As usual, if a set $\omega \subset \mathbb{R} \times \mathbb{R}^{n}$, then int $\omega$ and $\partial \omega$ denote the interior and the boundary of $\omega$, respectively.

Definition 1.1 (see [16]). Let the continuously differentiable functions $l_{i}(t, y), i=1,2, \ldots, p$ and $m_{j}(t, y), j=1,2, \ldots, q, p^{2}+q^{2}>0$ be defined on some open set $\omega_{0} \subset \mathbb{R} \times \mathbb{R}^{n}$. The set

$$
\omega^{*}=\left\{(t, y) \in \omega_{0}: l_{i}(t, y)<0, m_{j}(t, y)<0, i=1, \ldots, p, j=1, \ldots, q\right\}
$$

is called a regular polyfacial set with respect to the system (1.7), provided that it is nonempty, if $(\alpha)$ to $(\gamma)$ below hold.

$(\alpha)$ For $(t, \pi) \in \mathbb{R} \times C_{r}^{n}$ such that $(t+\theta, \pi(\theta)) \in \omega^{*}$ for $\theta \in[-r, 0)$, we have $(t, \pi) \in \Omega^{*}$.

( $\beta$ ) For all $i=1,2, \ldots, p$, all $(t, y) \in \partial \omega^{*}$ for which $l_{i}(t, y)=0$, and all $\pi \in C_{r}^{n}$ for which $\pi(0)=y$ and $(t+\theta, \pi(\theta)) \in \omega^{*}, \theta \in[-r, 0)$. It follows that $D l_{i}(t, y)>0$, where

$$
D l_{i}(t, y) \equiv \sum_{k=1}^{n} \frac{\partial l_{i}(t, y)}{\partial y_{k}} f_{k}(t, \pi)+\frac{\partial l_{i}(t, y)}{\partial t}
$$

$(\gamma)$ For all $j=1,2, \ldots, q$, all $(t, y) \in \partial \omega^{*}$ for which $m_{j}(t, y)=0$, and all $\pi \in C_{r}^{n}$ for which $\pi(0)=y$ and $(t+\theta, \pi(\theta)) \in \omega^{*}, \theta \in[-r, 0)$. It follows that $D m_{j}(t, y)<0$, where

$$
D m_{j}(t, y) \equiv \sum_{k=1}^{n} \frac{\partial m_{j}(t, y)}{\partial y_{k}} f_{k}(t, \pi)+\frac{\partial m_{j}(t, y)}{\partial t}
$$

The elements $(t, \pi) \in \mathbb{R} \times C_{r}^{n}$ in the sequel are assumed to be such that $(t, \pi) \in \Omega^{*}$.

In the following definition, a set $\omega^{*}$ is an arbitrary set without any connection with a regular polyfacial set $\omega^{*}$ defined by (1.9) in Definition 1.1. 
Definition 1.2. A system of initial functions $p_{A, \omega^{*}}$ with respect to the nonempty sets $A$ and $\omega^{*}$, where $A \subset \overline{\omega^{*}} \subset \mathbb{R} \times \mathbb{R}^{n}$ is defined as a continuous mapping $p: A \rightarrow C_{r}^{n}$ such that $(\alpha)$ and $(\beta)$ below hold.

( $\alpha$ ) If $z=(t, y) \in A \cap \operatorname{int} \omega^{*}$, then $(t+\theta, p(z)(\theta)) \in \omega^{*}$ for $\theta \in[-r, 0]$.

$(\beta)$ If $z=(t, y) \in A \cap \partial \omega^{*}$, then $(t+\theta, p(z)(\theta)) \in \omega^{*}$ for $\theta \in[-r, 0)$ and $(t, p(z)(0))=z$.

Definition 1.3 (see [17]). If $\mathcal{A} \subset B$ are subsets of a topological space and $\pi: B \rightarrow A$ is a continuous mapping from $B$ onto $\mathcal{A}$ such that $\pi(p)=p$ for every $p \in \mathcal{A}$, then $\pi$ is said to be a retraction of $B$ onto $A$. When a retraction of $B$ onto $A$ exists, $A$ is called a retract of $B$.

The following lemma describes the main result of the paper [16].

Lemma 1.4. Let $\omega^{*} \subset \omega_{0}$ be a regular polyfacial set with respect to the system (1.7), and let $W$ be defined as follows:

$$
W=\left\{(t, y) \in \partial \omega^{*}: m_{j}(t, y)<0, j=1,2, \ldots, q\right\}
$$

Let $Z \subset W \cup \omega^{*}$ be a given set such that $Z \cap W$ is a retract of $W$ but not a retract of $Z$. Then for each fixed system of initial functions $p_{Z, \omega^{*}}$, there is a point $z_{0}=\left(\sigma_{0}, y_{0}\right) \in Z \cap \omega^{*}$ such that for the corresponding solution $y\left(\sigma_{0}, p\left(z_{0}\right)\right)(t)$ of $(1.7)$, one has

$$
\left(t, y\left(\sigma_{0}, p\left(z_{0}\right)\right)(t)\right) \in \omega^{*}
$$

for each $t \in D_{\sigma_{0}, p\left(z_{0}\right)}$.

Remark 1.5. When Lemma 1.4 is applied, a lot of technical details should be fulfilled. In order to simplify necessary verifications, it is useful, without loss of generality, to vary the first coordinate $t$ in definition of the set $\omega^{*}$ in (1.9) within a half-open interval open at the right. Then the set $\omega^{*}$ is not open, but tracing the proof of Lemma 1.4, it is easy to see that for such sets it remains valid. Such possibility is used below. We will apply similar remark and explanation to sets of the type $\Omega, \Omega^{*}$ which serve as domains of definitions of functionals on the right-hand sides of equations considered.

For continuous vector functions

$$
\rho^{*}=\left(\rho_{1}^{*}, \rho_{2}^{*}, \ldots, \rho_{n}^{*}\right), \quad \delta^{*}=\left(\delta_{1}^{*}, \delta_{2}^{*}, \ldots, \delta_{n}^{*}\right):\left[t_{0}-r, \infty\right) \longrightarrow \mathbb{R}^{n}
$$

with $\rho^{*}(t) \ll \delta^{*}(t)$ for $t \in\left[t_{0}-r, \infty\right)$ (the symbol $\ll$ here and below means that $\rho_{i}^{*}(t)<\delta_{i}^{*}(t)$ for all $i=1,2, \ldots, n)$, continuously differentiable on $\left[t_{0}, \infty\right)$, we define the set

$$
\omega^{*}:=\left\{(t, y): t \in\left[t_{0}, \infty\right), \rho^{*}(t) \ll y \ll \delta^{*}(t)\right\}
$$

In the sequel, we employ the following result from [18, Theorem 1], which is proved with the aid of the retract technique combined with Razumikhin's approach. 
Theorem 1.6. Let there be a $p \in\{0, \ldots, n\}$ such that

(i) if $t \geq t_{0}, \phi \in \mathrm{C}_{r}^{n}$ and $(t+\theta, \phi(\theta)) \in \omega^{*}$ for any $\theta \in[-r, 0)$, then

$$
\begin{aligned}
& \left(\delta^{* i}\right)^{\prime}(t)<F^{i}(t, \phi), \text { when } \phi^{i}(0)=\delta^{* i}(t), \\
& \left(\rho^{* i}\right)^{\prime}(t)>F^{i}(t, \phi), \text { when } \phi^{i}(0)=\rho^{* i}(t)
\end{aligned}
$$

for any $i=1,2, \ldots, p$, (If $p=0$, this condition is omitted.)

(ii) if $t \geq t_{0}, \phi \in C_{r}^{n}$ and $(t+\theta, \phi(\theta)) \in \omega^{*}$ for any $\theta \in[-r, 0)$ then

$$
\begin{aligned}
& \left(\rho^{* i}\right)^{\prime}(t)<F^{i}(t, \phi), \text { when } \phi^{i}(0)=\rho^{* i}(t), \\
& \left(\delta^{* i}\right)^{\prime}(t)>F^{i}(t, \phi), \text { when } \phi^{i}(0)=\delta^{* i}(t)
\end{aligned}
$$

for any $i=p+1, p+2, \ldots, n$. (If $p=n$, this condition is omitted.) satisfies

Then, there exists an uncountable set $y$ of solutions of $(1.7)$ on $\left[t_{0}-r, \infty\right)$ such that each $y \in y$

$$
\rho^{*}(t) \ll y(t) \ll \delta^{*}(t), \quad t \in\left[t_{0}-r, \infty\right) .
$$

\subsection{Structure of Solutions of a Linear Equation}

In this section we focus our attention to structure of solutions of scalar linear differential equation of the type (1.3) with variable bounded delay of the form

$$
\dot{x}(t)=-c(t) x(t-\tau(t))
$$

with continuous functions $c:\left[t_{0}-r, \infty\right) \rightarrow \mathbb{R}^{+}$and $\tau:\left[t_{0}, \infty\right) \rightarrow(0, r]$.

In accordance with above definitions of positive or oscillatory solutions, we call a solution of (1.19) oscillatory if it has arbitrary large zeros, otherwise it is called nonoscillatory (positive or negative).

Let us mention properties of (1.19) which will be used later. Theorem 13 from [19] describes sufficient conditions for existence of positive solutions of (1.19) with nonzero limit.

Theorem 1.7 (see [19, Theorem 13]). Linear equation (1.19) has a positive solution with nonzero limit if and only if

$$
\int^{\infty} c(t) \mathrm{d} t<\infty
$$


Remark 1.8. Tracing the proof of Theorem 1.7, we conclude that a positive solution $x=x(t)$ of (1.19) with nonzero limit exists on $\left[t_{0}-r, \infty\right)$ if

$$
\int_{t_{0}-r}^{\infty} c(t) \mathrm{d} t<1
$$

The following theorem is a union of parts of results from [20] related to the structure formulas for solutions of (1.19).

Theorem 1.9. Suppose the existence of a positive solution of $(1.19)$ on $\left[t_{0}-r, \infty\right)$. Then there exist two positive solutions $x_{d}$ and $x_{s}$ of $(1.19)$ on $\left[t_{0}-r, \infty\right)$ satisfying the relation

$$
\lim _{t \rightarrow \infty} \frac{x_{s}(t)}{x_{d}(t)}=0
$$

such that every solution $x=x(t)$ of $(1.19)$ on $\left[t_{0}-r, \infty\right)$ can be represented by the formula

$$
x(t)=K x_{d}(t)+O\left(x_{s}(t)\right)
$$

where the constant $K$ depends on $x$.

The symbol $O$, applied in (1.23) and below, is the Landau order symbol frequently used in asymptotic analysis.

Moreover, Theorem 9 in [20] gives a possibility to replace the pair of solutions $x_{d}(t)$ and $x_{s}(t)$ in (1.23) by another pairs of solutions $\tilde{x}_{d}(t)$ and $\tilde{x}_{s}(t)$ if

$$
\lim _{t \rightarrow \infty} \frac{\tilde{x}_{s}(t)}{\tilde{x}_{d}(t)}=0
$$

as given in the following theorem.

Theorem 1.10. Let $\tilde{x}_{d}(t)$ and $\tilde{x}_{s}(t)$ be positive solutions of $(1.19)$ on $\left[t_{0}-r, \infty\right)$ such that $(1.24)$ holds. Then every solution $x=x(t)$ of $(1.19)$ on $\left[t_{0}-r, \infty\right)$ can be represented by the formula

$$
x(t)=K^{*} \tilde{x}_{d}(t)+O\left(\tilde{x}_{s}(t)\right),
$$

where the constant $K^{*}$ depends on $x$.

The next definition is based on the properties of solutions $x_{d}, \tilde{x}_{d}, x_{s}$, and $\tilde{x}_{s}$ described in Theorems 1.9 and 1.10 .

Definition 1.11 (see [20, Definition 2]). Suppose that the positive solutions $x_{d}$ and $x_{s}$ of (1.19) on $\left[t_{0}-r, \infty\right)$ satisfy the relation (1.22). Then, we call the solution $x_{d}$ a dominant solution and the solution $x_{s}$ a subdominant solution.

Due to linearity of (1.19), there are infinitely many dominant and subdominant solutions. Obviously, another pair of a dominant and a subdominant solutions is the pair $\tilde{x}_{d}(t), \tilde{x}_{s}(t)$ in Theorem 1.10 . 


\section{Main Results}

Let us consider two auxiliary linear equations:

$$
\begin{aligned}
& \dot{x}(t)=-C_{A}(t) x(t-r), \\
& \dot{z}(t)=-C_{B}(t) z(t-r),
\end{aligned}
$$

where $r \in \mathbb{R}^{+}$and $C_{A}, C_{B}$ are positive continuous functions on $\left[t_{0}-r, \infty\right), t_{0} \in \mathbb{R}$. According to the Theorems 1.7 and 1.9, both (2.1) and (2.2) have two types of positive solutions (subdominant and dominant). Let us denote them $x_{d}(t), x_{s}(t)$ for $(2.1)$ and $z_{d}(t), z_{s}(t)$ for (2.2), respectively, such that

$$
\lim _{t \rightarrow \infty} \frac{x_{s}(t)}{x_{d}(t)}=0, \quad \lim _{t \rightarrow \infty} \frac{z_{s}(t)}{z_{d}(t)}=0
$$

Without loss of generality, we can suppose that $x_{s}(t)<x_{d}(t)$ and $z_{s}(t)<z_{d}(t)$ on $\left[t_{0}-r, \infty\right)$.

\subsection{Auxiliary Linear Result}

The next lemma states that if $z_{d}(t), z_{s}(t)$ are dominant and subdominant solutions for (2.2), then there are dominant and subdominant solutions $x_{d}^{*}(t), x_{s}^{*}(t)$ for (2.1) satisfying certain inequalities.

Lemma 2.1. Let (1.5) be valid. Let $z_{d}(t), z_{s}(t)$ be dominant and subdominant solutions for (2.2). Then there are positive solutions $x_{s}^{*}(t), x_{d}^{*}(t)$ of $(2.1)$ on $\left[t_{0}-r, \infty\right)$ such that:
(a) $x_{s}^{*}(t)<z_{s}(t), t \in\left[t_{0}-r, \infty\right)$,
(b) $z_{d}(t)<x_{d}^{*}(t), t \in\left[t_{0}-r, \infty\right)$,
(c) $x_{d}^{*}(t)$ and $x_{s}^{*}(t)$ are dominant and subdominant solutions for (2.1).

Proof. (a) To prove the part (a), we employ Theorem 1.6 with $p=n=1$; that is, we apply the case (i). Consider (2.1), set $F(t, \phi):=-C_{A}(t) \phi(-r), \rho^{*}(t):=0, \delta^{*}(t):=z_{s}(t)$, and assume (see the case (i)):

$$
0<\phi(\theta)<z_{s}(t+\theta), \quad \theta \in[-r, 0), \phi(0)=z_{s}(t), t \geq t_{0} .
$$


Now we have to verify the inequalities (1.16), that is, in our case:

$$
\begin{aligned}
F(t, \phi)-\left(\delta^{*}\right)^{\prime}(t) & =-C_{A}(t) \phi(-r)-\left(\delta^{*}\right)^{\prime}(t) \\
& =-C_{A}(t) \phi(-r)-z_{S}^{\prime}(t) \\
& =-C_{A}(t) \phi(-r)+C_{B}(t) z_{S}(t-r) \\
& \geq \text { (we use }(1.5)) \\
& \geq-C_{B}(t) \phi(-r)+C_{B}(t) z_{S}(t-r) \\
& >C_{B}(t)\left[z_{S}(t-r)-z_{S}(t-r)\right]=0
\end{aligned}
$$

and $F(t, \phi)>\left(\delta^{*}\right)^{\prime}(t)$ if $t \in\left[t_{0}, \infty\right)$. Further, we have

$$
-F(t, \phi)+\left(\rho^{*}\right)^{\prime}(t)=C_{A}(t) \phi(-r)+0=C_{A}(t) \phi(-r)>0
$$

and $F(t, \phi)<\left(\rho^{*}\right)^{\prime}(t)$ if $t \in\left[t_{0}, \infty\right)$. Since both inequalities are fulfilled and all assumptions of Theorem 1.6 are satisfied for the case in question, there exists a solution $x_{s}^{*}(t)$ of (2.1) on $\left[t_{0}-r, \infty\right)$ such that $x_{s}^{*}(t)<z_{s}(t)$ for $t \in\left[t_{0}-r, \infty\right)$. problem:

(b) To prove the part (b), we consider a solution $x=x_{d}^{*}(t)$ of the following initial

$$
\begin{gathered}
\dot{x}(t)=-C_{A}(t) x(t-r), \quad t \in\left[t_{0}-r, \infty\right), \\
x(t)=z_{d}(t), \quad t \in\left[t_{0}-r, t_{0}\right] .
\end{gathered}
$$

Now, let us define a function

$$
W(t, x)=z_{d}(t)-x(t), \quad t \in\left[t_{0}-r, \infty\right)
$$

We find the sign of the full derivative of $W$ along the trajectories of (2.7) if $t \in\left[t_{0}, t_{0}+r\right]$ :

$$
\begin{aligned}
\left.\frac{\mathrm{d} W(t, x)}{\mathrm{d} t}\right|_{t \in\left[t_{0}, t_{0}+r\right]} & =-C_{B}(t) z_{d}(t-r)+C_{A}(t) x(t-r) \\
& =(\text { due to }(2.8)) \\
& =-C_{B}(t) z_{d}(t-r)+C_{A}(t) z_{d}(t-r) \\
& \leq\left[C_{A}(t)-C_{B}(t)\right] z_{d}(t-r) \leq(\text { due to }(1.5)) \leq 0 .
\end{aligned}
$$

It means that function $W$ is nonincreasing and it holds

$$
\begin{aligned}
W\left(t_{0}, x\left(t_{0}\right)\right) & =z_{d}\left(t_{0}\right)-x\left(t_{0}\right)=z_{d}\left(t_{0}\right)-z_{d}\left(t_{0}\right) \\
& =0 \geq W\left(t_{0}+\varepsilon, x\left(t_{0}+\varepsilon\right)\right)=z_{d}\left(t_{0}+\varepsilon\right)-x\left(t_{0}+\varepsilon\right), \quad \varepsilon \in[0, r],
\end{aligned}
$$


and hence $z_{d}\left(t_{0}+\varepsilon\right) \leq x\left(t_{0}+\varepsilon\right)$. It will be showed that this inequality holds also for every $t>t_{0}+r$.

On the contrary, let us suppose that the inequality is not true, that is, there exists a point $t=t^{* *}$ such that $z_{d}\left(t^{* *}\right)>x\left(t^{* *}\right)$. Then there exists a point $t^{*} \in\left[t_{0}, t^{* *}\right)$ such that $z_{d}\left(t^{*}\right)<x\left(t^{*}\right)$, otherwise $z_{d}(t) \equiv x(t)$ on $\left[t_{0}, t^{* *}\right]$. Without loss of generality, we can suppose that $x(t) \equiv z_{d}(t)$ on $\left[t_{0}, t^{* * *}\right]$ with a $t^{* * *} \in\left[t_{0}, t^{*}\right)$ and $x(t)>z_{d}(t)$ on $\left(t^{* * *}, t^{*}\right)$. Then, there exists a point $t^{\diamond} \in$ $\left(t^{* * *}, t^{*}\right)$ such that $x\left(t^{\diamond}\right)=K z_{d}\left(t^{\diamond}\right)$ for a constant $K>1$ and

$$
K z_{d}(t)>x(t), \quad \text { for } t \in\left[t_{0}, t^{\diamond}\right) .
$$

Hence, for a function $W^{*}(t, x)$ defined as $W^{*}(t, x):=K z_{d}(t)-x(t), t \in\left[t_{0}, t^{\diamond}\right]$, we get

$$
\begin{aligned}
\left.\frac{\mathrm{d} W^{*}(t, x)}{\mathrm{d} t}\right|_{t=t^{\circ}} & =K\left(-C_{B}(t) z_{d}(t-r)\right)+C_{A}(t) x(t-r) \\
& <(\text { due to }(2.12)) \\
& <K\left(-C_{B}(t) z_{d}(t-r)\right)+C_{A}(t) K z_{d}(t-r) \\
& =K z_{d}(t-r)\left[C_{A}(t)-C_{B}(t)\right] \leq(\text { by }(1.5)) \leq 0 .
\end{aligned}
$$

It means that $K z_{d}(t)<x(t)$ on a right-hand neighborhood of $t^{\diamond}$. This is a contradiction with inequality

$$
z_{d}(t)<K z_{d}(t)<x(t)
$$

hence it is proved that the existence of a solution $x_{d}^{*}(t)$ satisfies $z_{d}(t)<x_{d}^{*}(t)$ on $\left[t_{0}-r, \infty\right)$.

(c) To prove the part (c), we consider $\lim _{t \rightarrow \infty} x_{s}^{*}(t) / x_{d}^{*}(t)$. Due to (a) and (b), we get

$$
0 \leq \lim _{t \rightarrow \infty} \frac{x_{s}^{*}(t)}{x_{d}^{*}(t)} \leq \lim _{t \rightarrow \infty} \frac{z_{s}(t)}{z_{d}(t)}=0
$$

and $x_{d}^{*}(t)$ and $x_{s}^{*}(t)$ are (by Definition 1.11) dominant and subdominant solutions for (2.1).

\subsection{Existence of Positive Solutions of (1.3)}

The next theorems state that there exist two classes of positive solutions of (1.3) such that graphs of each solution of the first class are between graphs of dominant solutions of (2.1) and (2.2), and graphs of each solution of the second class are between graphs of subdominant solutions of (2.1) and (2.2), respectively. It means that we prove there are two classes of asymptotically different positive solutions of (1.3). Without loss of generality (see Remark 1.5), we put $\Omega:=\left[t_{0}, \infty\right) \times C_{r}^{1}$. In the following, we will use our main supposition (1.4); that is, we assume that for $(t, \phi) \in \Omega$ inequalities,

$$
C_{A}(t) \phi(-r) \leq f(t, \phi) \leq C_{B}(t) \phi(-r)
$$

hold, where $\phi$ is supposed to be positive. 
Theorem 2.2. Let $f: \Omega \rightarrow \mathbb{R}$ be a continuous quasibounded functional. Let inequality (1.5) be valid, and (2.16) holds for any $(t, \phi) \in \Omega$ with $\phi(\theta)>0, \theta \in[-r, 0]$. Let $x(t)$ be a positive solution of (2.1) on $\left[t_{0}-r, \infty\right)$, and let $z(t)$ be a positive solution of $(2.2)$ on $\left[t_{0}-r, \infty\right)$ such that $x(t)<z(t)$ on $\left[t_{0}-r, \infty\right)$. Then there exists an uncountable set $y$ of positive solutions of $(1.3)$ on $\left[t_{0}-r, \infty\right)$ such that each solution $y \in y$ satisfies

$$
x(t)<y(t)<z(t)
$$

for $t \in\left[t_{0}-r, \infty\right)$.

Proof. To prove this theorem, we employ Theorem 1.6 with $p=n=1$; that is, we apply the case (i). Set $F\left(t, y_{t}\right):=-f\left(t, y_{t}\right), \rho^{*}(t):=x(t), \delta^{*}(t):=z(t)$; hence, the set $\omega^{*}$ will be defined as

$$
\omega^{*}:=\left\{(t, y): t \in\left[t_{0}-r, \infty\right), x(t)<y(t)<z(t)\right\}
$$

Now, we have to verify the inequalities (1.16). In our case

$$
\begin{aligned}
F(t, \phi)-\left(\delta^{*}\right)^{\prime}(t) & =-f(t, \phi)-\left(\delta^{*}\right)^{\prime}(t) \\
& =-f(t, \phi)-z^{\prime}(t) \\
& =-f(t, \phi)+C_{B}(t) z(t-r) \\
& \geq(\text { we use }(2.16)) \\
& \geq-C_{B}(t) \phi(-r)+C_{B}(t) z(t-r) \\
& >(\text { we use }(2.18): \phi(-r)<z(t-r)) \\
& >C_{B}(t)[z(t-r)-z(t-r)]=0, \\
-F(t, \phi)+\left(\rho^{*}\right)^{\prime}(t) & =f(t, \phi)+\left(\rho^{*}\right)^{\prime}(t) \\
& =f(t, \phi)+x^{\prime}(t) \\
& =f(t, \phi)-C_{A}(t) x(t-r) \\
& \geq(\text { we use }(2.16)) \\
& \geq C_{A}(t) \phi(-r)-C_{A}(t) x(t-r) \\
& >(\text { we use }(2.18): \phi(-r)>x(t-r)) \\
& >C_{A}(t)[x(t-r)-x(t-r)]=0 .
\end{aligned}
$$

Therefore,

$$
\begin{gathered}
F(t, \phi)-\left(\delta^{*}\right)^{\prime}(t)>0, \\
-F(t, \phi)+\left(\rho^{*}\right)^{\prime}(t)>0 .
\end{gathered}
$$


Both inequalities (1.16) are fulfilled, and all assumptions of Theorem 1.6 are satisfied for the case in question. There exists class of positive solutions $y$ of $(1.3)$ on $\left[t_{0}-r, \infty\right)$ that for each solution $y \in y$ from this class it is satisfied that $x(t)<y(t)<z(t)$ for $t \in\left[t_{0}-r, \infty\right)$.

Corollary 2.3. Let, in accordance with Lemma 2.1, $x_{S}(t)$ be the subdominant solution of (2.1), and let $z_{s}(t)$ be the subdominant solution of $(2.2)$, that is, $x_{s}(t)<z_{s}(t)$ on $\left[t_{0}-r, \infty\right)$. Then, there exists an uncountable set $y_{s}$ of positive solutions of $(1.3)$ on $\left[t_{0}-r, \infty\right)$ such that each solution $y_{s} \in y_{s}$ satisfies

$$
x_{s}(t)<y_{s}(t)<z_{s}(t)
$$

If inequality (1.6) holds, then dominant solutions $x_{d}(t)$ of (2.1) and $z_{d}(t)$ of (2.2) have finite positive limits

$$
\begin{aligned}
& C_{x}:=\lim _{t \rightarrow \infty} x_{d}(t), \quad C_{x}>0, \\
& C_{z}:=\lim _{t \rightarrow \infty} z_{d}(t), \quad C_{z}>0 .
\end{aligned}
$$

This is a simple consequence of positivity of solutions $x_{d}(t), z_{d}(t)$ and properties of dominant and subdominant solutions (see Theorem 1.7, Remark 1.8, Theorem 1.9, formulas (1.22)(1.25) and (2.3)). Then, due to linearity of (2.1) and (2.2), it is clear that there are dominant solutions $x_{d}(t), z_{d}(t)$ of both equations such that $z_{d}(t)<x_{d}(t)$ on $\left[t_{0}-r, \infty\right)$. In the following lemma, we without loss of generality suppose that $x_{d}(t)$ and $z_{d}(t)$ are such solutions and their initial functions are nonincreasing on initial interval $\left[t_{0}-r, t_{0}\right]$. We will need constants $M$ and $L$ satisfying

$$
\begin{aligned}
& M>M^{*}:=\frac{x_{d}\left(t_{0}-r\right)}{C_{z}}, \\
& L>L^{*}:=\frac{M z_{d}\left(t_{0}-r\right)}{C_{x}} .
\end{aligned}
$$

Lemma 2.4. Let $f: \Omega \rightarrow \mathbb{R}$ be a continuous quasibounded functional. Let inequalities (1.5) and (1.6) be valid, and (2.16) holds for any $(t, \phi) \in \Omega$ with $\phi(\theta)>0, \theta \in[-r, 0]$. Let $x_{d}(t), t \in\left[t_{0}-r, \infty\right)$ be a dominant solution of (2.1), nonincreasing on $\left[t_{0}-r, t_{0}\right]$, and let $z_{d}(t), t \in\left[t_{0}-r, \infty\right)$ be a dominant solution of (2.2), nonincreasing on $\left[t_{0}-r, t_{0}\right]$, such that $z_{d}(t)<x_{d}(t), t \in\left[t_{0}-r, \infty\right)$. Then there exists another dominant solution $z_{d}^{*}(t)$ of $(2.2)$ and a positive solution $y=y_{d}(t)$ of $(1.3)$ on $\left[t_{0}-r, \infty\right)$ such that it holds that

$$
x_{d}(t)<y_{d}(t)<z_{d}^{*}(t)
$$

for $t \in\left[t_{0}-r, \infty\right)$ and $z_{d}^{*}(t)=M z_{d}(t)$. 
Proof. Both dominant solutions $x_{d}(t)$ and $z_{d}(t)$, of (2.1) and (2.2), respectively, have nonzero positive limits $C_{x}$ and $C_{z}$. From linearity of (2.1) and (2.2), it follows that solutions multiplied by an arbitrary constant are also solutions of (2.1) and (2.2), respectively. It holds that

$$
z_{d}^{*}\left(t_{0}-r\right)=M z_{d}\left(t_{0}-r\right) \geq M z_{d}(t)=z_{d}^{*}(t)>M C_{z}>x_{d}\left(t_{0}-r\right) \geq x_{d}(t),
$$

where $t \in\left[t_{0}-r, \infty\right)$.

Now, we define the set $\omega^{*}$ in the same way as (2.18) in the proof of Theorem 2.2, but with $x_{d}(t)$ instead of $x(t)$ and with $z_{d}^{*}(t)$ instead of $z(t)$, that is,

$$
\omega^{*}:=\left\{(t, y): t \in\left[t_{0}-r, \infty\right), x_{d}(t)<y(t)<z_{d}^{*}(t)\right\}
$$

According to the Theorem 2.2 (with $x_{d}(t)$ instead of $x(t)$ and with $z_{d}^{*}(t)$ instead of $z(t)$ ), it is visible that there exists a positive solution $y=y_{d}(t)$ of (1.3) satisfying

$$
x_{d}(t)<y_{d}(t)<z_{d}^{*}(t),
$$

where $t \in\left[t_{0}, \infty\right)$; that is, inequalities (2.24) hold.

Theorem 2.5. Let all suppositions of Lemma 2.4 be valid, and let $y_{d}(t)$ be a solution of (1.3) satisfying inequalities (2.24). Then, there exists a positive solution $x_{d}^{* *}(t)$ of $(2.1)$ on $\left[t_{0}-r, \infty\right)$ satisfying

$$
z_{d}(t)<y_{d}(t)<x_{d}^{* *}(t)
$$

where $x_{d}^{* *}(t)=L x_{d}(t)$ and $t \in\left[t_{0}-r, \infty\right)$.

Proof. Multiplying solution $x_{d}(t)$ by the constant $L$, we have

$$
L x_{d}(t)>L C_{x}>M z_{d}\left(t_{0}-r\right)
$$

Using (2.29) and (2.24), we get

$$
x_{d}^{* *}(t)=L x_{d}(t)>M z_{d}\left(t_{0}-r\right)=z_{d}^{*}\left(t_{0}-r\right)>z_{d}^{*}(t)>y_{d}(t)>x_{d}(t)>z_{d}(t),
$$

where $t \in\left[t_{0}-r, \infty\right)$. Hence, there exists a solution $y_{d}(t)$ of (1.3) such that inequalities (2.28) hold.

\subsection{Asymptotically Different Behavior of Positive Solutions of (1.3)}

Somewhat reformulating the statement of Theorem 2.5, we can define a class of positive solutions $y_{d}$ of (1.3) such that every solution $y_{d} \in y_{d}$ is defined on $\left[t_{0}-r, \infty\right)$ and satisfies

$$
C z_{d}(t)<y_{d}(t)<C x_{d}^{* *}(t),
$$


where $t \in\left[t_{0}-r, \infty\right)$ for a positive constant $C$ and, for every positive constant $C$, there exists a solution $y_{d} \in y_{d}$ satisfying $(2.31)$ on $\left[t_{0}-r, \infty\right)$.

The following theorem states that positive solutions $y_{s}(t)$ and $y_{d}(t)$ of (1.3) have a different order of vanishing.

Theorem 2.6. Let all the assumptions of Corollary 2.3 and Theorem 2.5 be met. Then there exist two classes $y_{s}$ and $y_{d}$ of positive solutions of (1.3) described by inequalities (2.21) and (2.31). Every two solutions $y_{s}, y_{d}$, such that $y_{s} \in y_{s}$ and $y_{d} \in y_{d}$, have asymptotically different behavior, that is,

$$
\lim _{t \rightarrow+\infty} \frac{y_{s}(t)}{y_{d}(t)}=0
$$

Proof. Let the solution $y_{s}(t)$ be the one specified in Corollary 2.3 and the solution $y_{d}(t)$ specified by (2.31) with a positive constant $C$. Now let us verify that (2.32) holds. With the aid of inequalities (2.21) and (2.31), we get

$$
0 \leq \lim _{t \rightarrow+\infty} \frac{y_{s}(t)}{y_{d}(t)} \leq \lim _{t \rightarrow+\infty} \frac{z_{s}(t)}{C z_{d}(t)}=0
$$

in accordance with (1.22), since $z_{s}(t)$ and $z_{d}(t)$ are positive (subdominant and dominant) solutions of linear equation (2.2).

Another final statement, being a consequence of Lemma 2.1 and Theorems 2.2 and 2.5, is the following.

Theorem 2.7. Let $f: \Omega \rightarrow \mathbb{R}$ be a continuous quasibounded functional. Let inequalities (1.5) and (1.6) be valid, and (2.16) holds for any $(t, \phi) \in \Omega$ with $\phi(\theta)>0, \theta \in[-r, 0]$. Then on $\left[t_{0}-r, \infty\right)$ there exist

(a) dominant and subdominant solutions $x_{d}(t), x_{s}(t)$ of (2.1),

(b) dominant and subdominant solutions $z_{d}(t), z_{s}(t)$ of (2.2),

(c) solutions $y_{d}(t), y_{s}(t)$ of (1.3)

such that

$$
\begin{gathered}
0<x_{S}(t)<y_{s}(t)<z_{s}(t)<z_{d}(t)<y_{d}(t)<x_{d}(t), \\
\lim _{t \rightarrow \infty} \frac{x_{s}(t)}{x_{d}(t)}=\lim _{t \rightarrow \infty} \frac{z_{s}(t)}{z_{d}(t)}=\lim _{t \rightarrow \infty} \frac{y_{s}(t)}{y_{d}(t)}=0 .
\end{gathered}
$$

Example 2.8. Let (1.3) be reduced to

$$
\dot{y}(t)=-f\left(t, y_{t}\right):=-3 t \exp \left(-3 t+\frac{1}{2} \cos (t y(t-1))\right) \cdot y(t-1),
$$


and let auxiliary linear equations (2.1) and (2.2) be reduced to

$$
\begin{aligned}
& \dot{x}(t)=-4 t \exp (2-4 t) \cdot x(t-1), \\
& \dot{z}(t)=-2 t \exp (1-2 t) \cdot z(t-1),
\end{aligned}
$$

that is,

$$
C_{A}(t):=4 t \exp (2-4 t), \quad C_{B}(t):=2 t \exp (1-2 t), \quad r=1 .
$$

Let $t_{0}$ be sufficiently large. Inequalities (1.5), (1.6), and (2.16) hold. In view of linearity and by Remark 1.8, we conclude that there exist dominant solutions $x_{d}(t)$ of $(2.37)$ and $z_{d}(t)$ of (2.38) such that

$$
\lim _{t \rightarrow \infty} x_{d}(t)=11, \quad \lim _{t \rightarrow \infty} z_{d}(t)=2, \quad z_{d}(t)<x_{d}(t), t \in\left[t_{0}-1, \infty\right)
$$

Moreover, there exist subdominant solutions $x_{s}(t)$ of (2.37) and $z_{s}(t)$ of (2.38) such that $x_{s}(t)<z_{s}(t), t \in\left[t_{0}-1, \infty\right)$ which are defined as

$$
x_{S}(t):=\exp \left(-2 t^{2}\right), \quad z_{s}(t):=\exp \left(-t^{2}\right)
$$

By Theorem 2.7, we conclude that there exist solutions $y_{s}(t)$ and $y_{d}(t)$ of (2.36) satisfying inequalities (2.34), and (without loss of generality) inequalities

$$
0<x_{s}(t)=\exp \left(-2 t^{2}\right)<y_{s}(t)<z_{s}(t)=\exp \left(-t^{2}\right)<1 \leq z_{d}(t)<y_{d}(t)<10 \leq x_{d}(t)
$$

hold on $\left[t_{0}-1, \infty\right)$.

\section{Conclusions and Open Problems}

The following problems were not answered in the paper and present interesting topics for investigation.

Open Problem 3.1. In Lemma 2.4 and Theorems 2.5-2.7 we used the convergence assumption (1.6) being, without loss of generality, equivalent to

$$
\int^{\infty} C_{B}(t) \mathrm{d} t<\infty
$$

It is an open question whether similar results could be proved if the integral is divergent, that is, if

$$
\int^{\infty} C_{B}(t) \mathrm{d} t=\infty .
$$


Open Problem 3.2. Dominant and subdominant solutions are used for representation of family of all solutions of scalar linear differential delayed equation, for example, by formula (1.25). Investigation in this line of the role of solutions $y_{d}(t)$ and $y_{s}(t)$ of (1.3) (see Theorems 2.6 and 2.7) is an important question. Namely, it seems to be an interesting question to establish sufficient conditions for the right-hand side of (1.3) such that its every solution $y=y(t)$ can be represented on $\left[t_{0}-r, \infty\right)$ by the formula

$$
y(t)=K y_{d}(t)+O\left(y_{s}(t)\right)
$$

where the constant $K$ depends only on $y(t)$.

Open Problem 3.3. The notions dominant and subdominant solutions are in the cited papers defined for scalar differential delayed equations only. It is a rather interesting question if the results presented can be enlarged to systems of differential delayed equations.

Remark 3.4. Except for papers and books mentioned in this paper we refer, for example, to sources [21-23], treating related problems as well. Note that the topic is connected with similar questions for discrete equations (e.g., [24-27]).

\section{Acknowledgments}

This research was supported by the Grant no. 1/0090/09 of the Grant Agency of Slovak Republic (VEGA) and by the Project APVV-0700-07 of Slovak Research and Development Agency.

\section{References}

[1] R. P. Agarwal, M. Bohner, and W.-T. Li, Nonoscillation and Oscillation: Theory for Functional Differential Equations, vol. 267 of Monographs and Textbooks in Pure and Applied Mathematics, Marcel Dekker, New York, NY, USA, 2004.

[2] R. D. Driver, Ordinary and Delay Differential Equations, Springer, New York, NY, USA, 1977.

[3] L. H. Erbe, Q. Kong, and B. G. Zhang, Oscillation Theory for Functional-Differential Equations, vol. 190 of Monographs and Textbooks in Pure and Applied Mathematics, Marcel Dekker, New York, NY, USA, 1995.

[4] K. Gopalsamy, Stability and Oscillations in Delay Differential Equations of Population Dynamics, vol. 74 of Mathematics and Its Applications, Kluwer Academic Publishers, Dordrecht, The Netherlands, 1992.

[5] I. Győri and G. Ladas, Oscillation Theory of Delay Differential Equations, Oxford Mathematical Monographs, The Clarendon Press Oxford University Press, New York, NY, USA, 1991.

[6] V. Kolmanovskiu and A. Myshkis, Applied Theory of Functional-Differential Equations, vol. 85 of Mathematics and Its Applications (Soviet Series), Kluwer Academic Publishers, Dordrecht, The Netherlands, 1992.

[7] B. Dorociaková and R. Olach, "Existence of positive solutions of delay differential equations," Tatra Mountains Mathematical Publications, vol. 43, pp. 63-70, 2009.

[8] E. Kozakiewicz, “Über das asymptotische Verhalten der nichtschwingenden Lösungen einer linearen Differentialgleichung mit nacheilendem Argument," Wissenschaftliche Zeitschrift Der Humboldt Universitat Zu Berlin, Mathematisch-Naturwissenschaftliche Reihe, vol. 13, no. 4, pp. 577-589, 1964.

[9] E. Kozakiewicz, "Zur Abschätzung des Abklingens der nichtschwingenden Lösungen einer linearen Differentialgleichung mit nacheilendem Argument," Wissenschaftliche Zeitschrift Der Humboldt Universitat Zu Berlin, Mathematisch-Naturwissenschaftliche Reihe, vol. 15, pp. 675-676, 1966.

[10] E. Kozakiewicz, "Über die nichtschwingenden Lösungen einer linearen Differentialgleichung mit nacheilendem Argument," Mathematische Nachrichten, vol. 32, pp. 107-113, 1966. 
[11] M. Pituk, "Special solutions of functional differential equations," Studies of the University of Žilina. Mathematical Series, vol. 17, no. 1, pp. 115-122, 2003.

[12] Yu. A. Ryabov, "Certain asymptotic properties of linear systems with small time lag," Trudy Sem. Teor. Diff. Uravnenii s Otklon. Argumentom Univ. Druzby Narodov Patrisa Lumumby, vol. 3, pp. 153-165, 1965 (Russian).

[13] J. Diblík and M. Kúdelčíková, "Two classes of asymptotically different positive solutions of the equation $\dot{y}(t)=-f\left(t, y_{t}\right)$," Nonlinear Analysis. Theory, Methods $\mathcal{E}$ Applications, vol. 70, no. 10, pp. 37023714, 2009.

[14] J. K. Hale and S. M. Verduyn Lunel, Introduction to Functional-Differential Equations, vol. 99 of Applied Mathematical Sciences, Springer, New York, NY, USA, 1993.

[15] T. Ważewski, "Sur un principe topologique de l'examen de l'allure asymptotique des intégrales des équations différentielles ordinaires," Annales De La Societe Polonaise De Mathematique, vol. 20, pp. 279_ 313, 19487.

[16] K. P. Rybakowski, "Ważewski's principle for retarded functional differential equations," Journal of Differential Equations, vol. 36, no. 1, pp. 117-138, 1980.

[17] V. Lakshmikantham and S. Leela, Differential and Integral Inequalities-vol. I. Ordinary Differential Equations, Academic Press, New York, NY, USA, 1969.

[18] J. Diblík, "A criterion for existence of positive solutions of systems of retarded functional-differential equations," Nonlinear Analysis. Theory, Methods \& Applications, vol. 38, no. 3, pp. 327-339, 1999.

[19] J. Diblík, "Behaviour of solutions of linear differential equations with delay," Archivum Mathematicum, vol. 34, no. 1, pp. 31-47, 1998.

[20] J. Diblík and M. Růžičková, "Asymptotic behavior of solutions and positive solutions of differential delayed equations," Functional Differential Equations, vol. 14, no. 1, pp. 83-105, 2007.

[21] J. Čermák, "On a linear differential equation with a proportional delay," Mathematische Nachrichten, vol. 280, no. 5-6, pp. 495-504, 2007.

[22] I. Culáková, L. Hanuštiaková, and R. Olach, "Existence for positive solutions of second-order neutral nonlinear differential equations," Applied Mathematics Letters, vol. 22, no. 7, pp. 1007-1010, 2009.

[23] L. K. Kikina and I. P. Stavroulakis, "A survey on the oscillation of solutions of first order delay difference equations," Cubo, vol. 7, no. 2, pp. 223-236, 2005.

[24] J. Baštinec and J. Diblík, "Subdominant positive solutions of the discrete equation $\Delta u(k+n)=$ - $p(k) u(k), "$ Abstract and Applied Analysis, vol. 2004, pp. 461-470, 2004.

[25] L. Berezansky, E. Braverman, and O. Kravets, "Nonoscillation of linear delay difference equations with positive and negative coefficients," Journal of Difference Equations and Applications, vol. 14, no. 5, pp. 495-511, 2008.

[26] I. Győri and L. Horváth, "Asymptotic constancy in linear difference equations: limit formulae and sharp conditions," Advances in Difference Equations, vol. 2010, Article ID 789302, 20 pages, 2010.

[27] S. Stević and K. S. Berenhaut, "The behavior of positive solutions of a nonlinear second-order difference equation," Abstract and Applied Analysis, vol. 2008, Article ID 653243, 8 pages, 2008. 


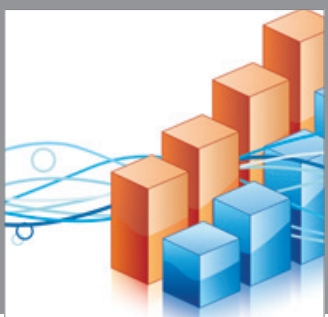

Advances in

Operations Research

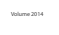

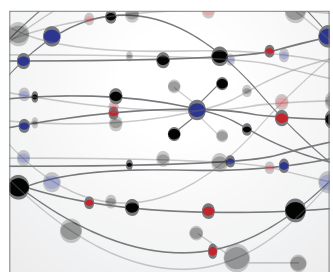

\section{The Scientific} World Journal
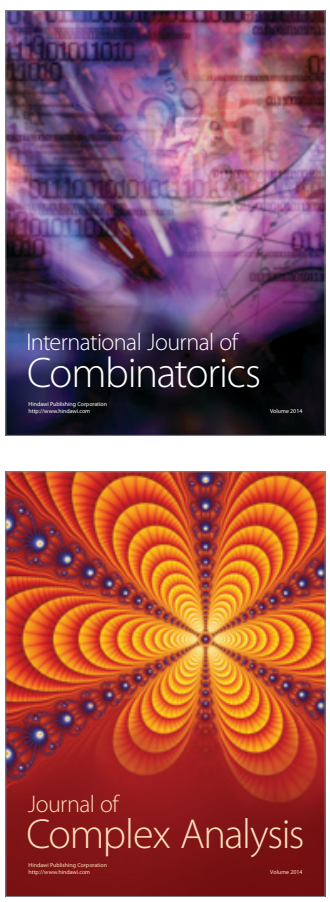

International Journal of

Mathematics and

Mathematical

Sciences
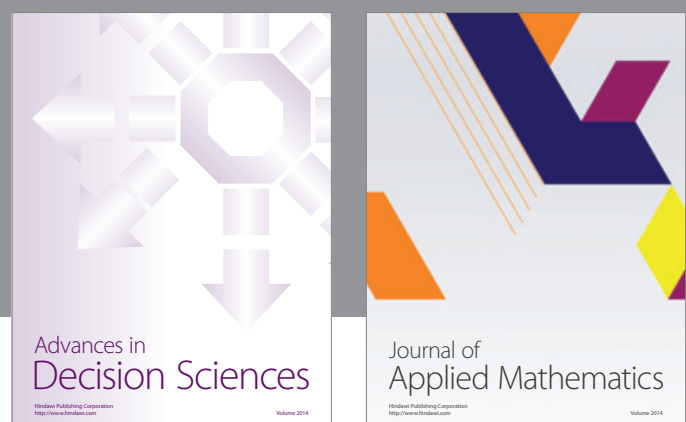

Journal of

Applied Mathematics
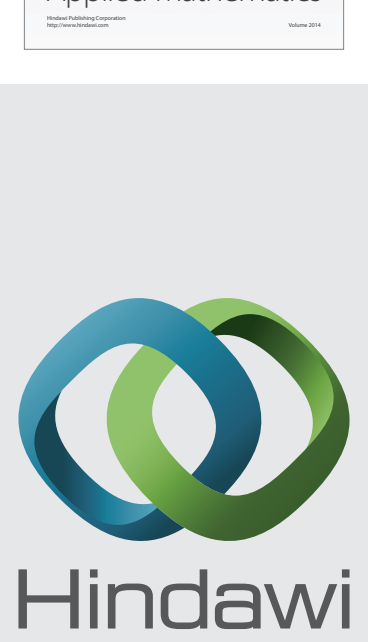

Submit your manuscripts at http://www.hindawi.com
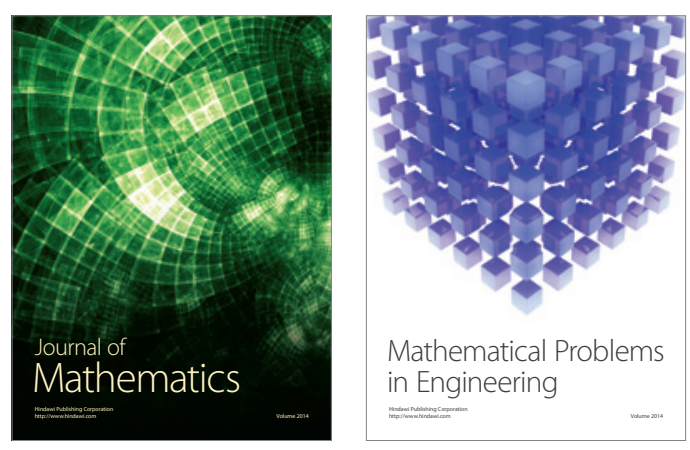

Mathematical Problems in Engineering
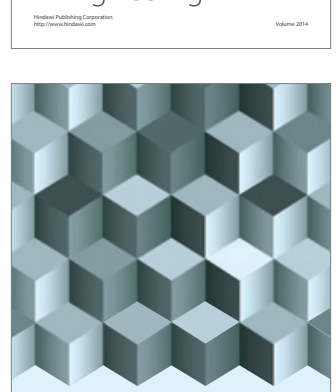

Journal of

Function Spaces
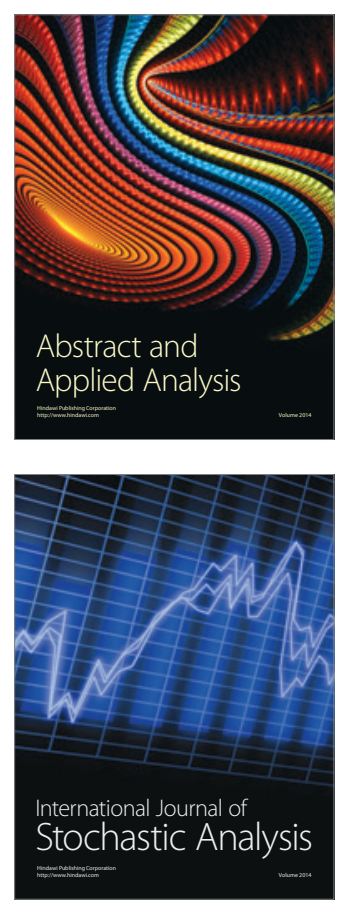

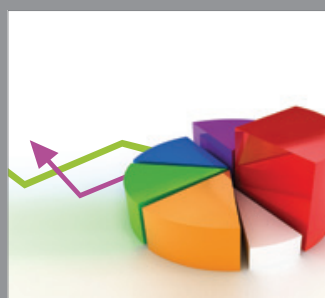

ournal of

Probability and Statistics

Promensencen
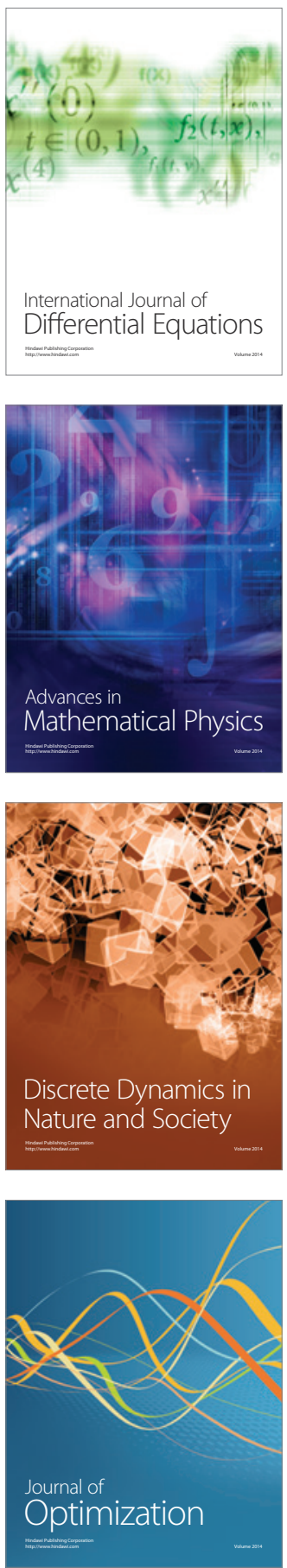